\title{
Detection of Minimal Residual Disease
}

National Cancer Institute

\section{Source}

National Cancer Institute. Detection of Minimal Residual Disease. NCI Thesaurus. Code C15795.

The discovery of a tumor or cancer after primary, potentially curative therapy. 